\title{
Prolonged grief proposed as mental disorder
}

I $\mathrm{n}$ the field of mental health, the line between well and ill is not always clear. But that line can pretty much disappear, taking "well" along with it, when a new diagnosis appears in the Diagnostic and Statistical Manual of Mental Disorders (DSM). What was once an emotion becomes a symptom. What was once something to be endured becomes something to be treated. This may soon be the case for people who grieve intensely for prolonged periods.

The next edition of the manual $D S M-5$, scheduled for publication in 2013 - is likely to contain a new entry called bereavement-related disorder. It had previously been proposed for DSM$I V$, published in 1994, though the supporting evidence was lacking at the time. Since then, however, research in this area has grown immensely, and this time the diagnosis is likely to gain entry, says Dr. Katherine Shear, Marion E. Kenworthy Professor of Psychiatry at the Columbia University School of Social Work in New York City, New York.

The proposed disorder goes by different names in scientific literature: prolonged grief disorder, complex grief, traumatic grief, pathological grief and, perhaps most commonly, complicated grief. It also means different things depending on whom you ask, but in general it applies to people whose lives are considerably impaired for longerthan-normal periods after the loss of a loved one. Shear supports the addition of this disorder to the DSM, saying it will benefit patients.

"It's striking, when you work in this area, to see how it helps patients enormously to know what is wrong with them," she says.

In a recent paper, Shear and several colleagues considered whether complicated grief met the criteria for a treatable mental disorder (Depress Anxiety 2011;28:103-17). They suggest that about $10 \%$ of bereaved people develop complicated grief, and that inordinately prolonged symptoms - persistent feelings of disbelief and anger, a sense of

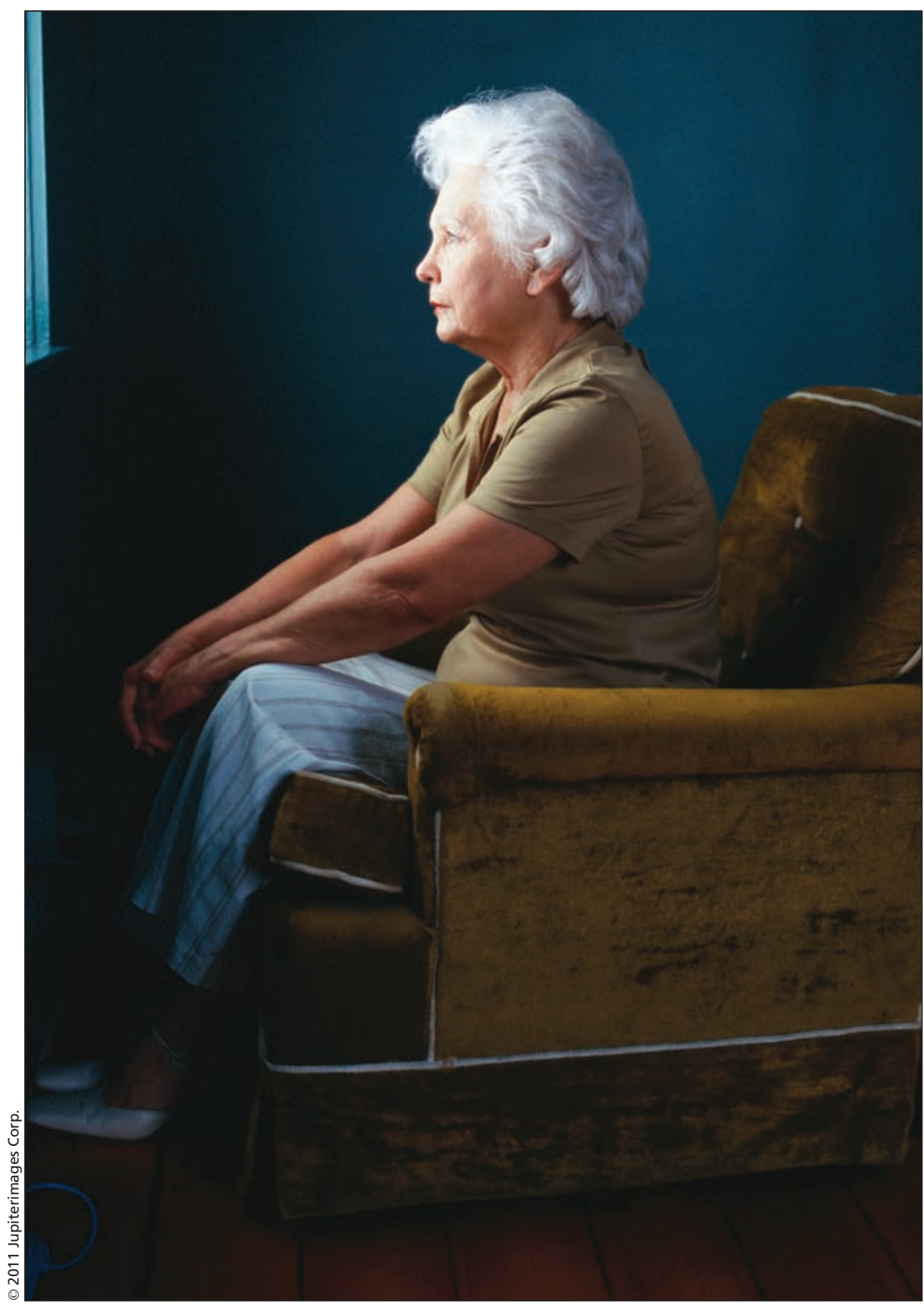

Bereaved individuals whose lives are considerably impaired for prolonged periods after the death of a loved one may soon be formally diagnosed as having a mental disorder that requires treatment.

emptiness, suicidal thoughts, estrangement from other people - warrant a targeted treatment.

"It is now clear that grief can be complicated, much as wound healing can be complicated, such that intensity of symptoms is heightened and their duration prolonged," states the paper. "We conclude that a new category of complicated grief is needed in DSM-5 and suggest that the management of bereaved people can be improved by this and other modifications in DSM-5." 
One of the primary advantages of adding complicated grief to the $D S M$ is that it will spur the development of treatments, says Shear. In some cases, the natural healing process can only begin after the complications have been dealt with.

Of course, the addition of complicated grief to the DSM will not make a difference in the lives of most bereaved people. Even with the hardest losses, says Shear, most people are resilient and don't experience prolonged grief. But certain people will, including those with a history of mood or anxiety disorders. An untoward death, such as one due to an easily preventable medical error, may also complicate the grieving process. It also depends, naturally, on the relationship one has with the deceased.

"People have an intrinsic need to form and maintain close relationships with a small number of people," says Shear. "Complicated grief can occur when you lose one of those people."

Even if certain people are predisposed to process grief more poorly than others, that doesn't necessarily mean that adding it to the DSM is a good thing, says Leeat Granek, who has a $\mathrm{PhD}$ in psychology and is now a postdoctoral fellow at McMcaster University in Hamilton, Ontario.

"In our culture, if you are not over a loss almost immediately, a couple of weeks or a few months, you are made to feel like something is wrong with you," says Granek. "A diagnosis will formalize that and make it even easier for people to show little tolerance for grief in everyday life."

In a paper exploring the evolution of grief theory in psychology, Granek suggested that grief is viewed by modern psychologists as something that must be recovered from quickly and completely (Hist Psychol 2010;13:46-73). People who don't respond to grief in this way are therefore viewed as in need of psychological intervention, which, the paper suggests, points to a larger trend of pathologizing anything that causes distress: "The pathologization of grief is part of a widespread phenomena of turning everyday problems into psychological disorders to be managed and treated by mental health professionals."

Granek is not alone in her concerns about the proliferation of mental disorders. Some mental health professionals question such newly formed disorders as Internet addiction (www.cmaj.ca/cgi /doi/10.1503/cmaj.109-3052). If every mental health professional's pet condition entered the DSM, some suggest, it will cease being a useful document (www.cmaj.ca/cgi/doi/10.1503/cmaj.10 9-3105).

Even when most mental health professionals agree that something is a pathological condition, figuring out who has that condition will vary from one clinician to another, says Granek. For complicated grief, some base a diagnosis on longevity while others focus on severity. Some say it is very prevalent and some say it is rare.

"All the infighting is about criteria and who gets to say what is normal and what isn't normal," says Granek. "There's something arbitrary about that."

Instead of making grief an illness, society would be better served by opening a public dialogue about grief and by encouraging people to seek help in their communities, not in the private offices of psychotherapists, says Granek. There should be more education on grief in schools so children are better equipped to understand and talk about loss. Our culture needs to learn that grief is not always something people "get over," she says. A loss of a child, for instance, can be lifechanging, creating a "new normal" for grieving parents.

"The thing about grief is that every single person is going to grieve at some point in their life," says Granek. "When you take something that is a normal part of everyday life and pathologize it, it can have a powerful impact on how people view it." - Roger Collier, CMAJ

CMAJ 2011. DOI:10.1503/cmaj.109-3819 